\title{
Management of Esophageal Perforation Secondary to Dilation by Peptic Stenosis in a General Hospital of Lima-peru
}

\section{Castillo De La Cadena Luis*}

Hospital Nacional María Auxiliadora, Lima -Perú, Peru

*Corresponding Author: Castillo De La Cadena Luis. E-mail: medicoluis@gmail.com
Received: October 18, 2021

Published: November 23, 2021

(C) All rights are reserved by Castillo De La Cadena Luis.

\begin{abstract}
Esophageal perforation secondary to peptic stricture dilatation is a potential serious complication secondary to endoscopic procedures. There is no consensus about the management of esophageal perforation, especially in those secondary to peptic stricture dilatation.

Esophageal perforation is a not very frequent high mortality pathology. It is of interest to present the multidisciplinary and timely management of a patient with esophageal perforation secondary to peptic stricture dilatation in a general hospital in Lima.
\end{abstract}

Keywords: Esophageal Stricture; Surgery; Chest Pain; Dysphagia

\section{Introduction}

Esophageal perforation secondary to peptic stricture dilatation is a rare condition that is associated with high mortality [1,2]. It is a potential serious complication secondary to endoscopic procedures, especially in esophageal dilatations (0.03 - 0.5\%).

Even with rapid diagnosis and optimal treatment, mortality is significant and has consistently ranged from 15 to $20 \%$ [2].

There is no consensus about the management of esophageal perforation, especially in those secondary to peptic stricture dilatation $[3,4]$.

Esophageal perforation produces communication of oral and gastric secretions to the mediastinum, with the consequent intense and rapidly progressive inflammatory process. This process quickly oversomes the body's defensive capacity, leading to lifethreatening effects.
To reduce morbidity and mortality, rapid diagnosis, contamination elimination, infection control, and critical care monitoring are required [1-4].

Esophageal perforation is a not very frequent high mortality pathology. It is of interest to present the multidisciplinary and timely management of a patient with esophageal perforation secondary to peptic stricture dilatation in a general hospital in Lima.

\section{Clinical Case}

61-year-old woman, native and from Cañete, Lima, is referred to the Emergency Department of the Gastroenterology procedures area of the María Auxiliadora Hospital, due to suspicion of esophageal perforation secondary to peptic stricture dilatation. Upon admission, the patient reported pain at the cervical level and pain in the anterior superior aspect of the thorax. In addition, the patient presented pain when swallowing saliva. 
At the entrance examination, a BP: $90 / 60 \mathrm{mmHg}$, HR: $100 \mathrm{bpm}$, T: $37^{\circ} \mathrm{C}$, Weight: $62 \mathrm{Kg} . \mathrm{O}_{2}$ respiratory rate of 20 breaths $/ \mathrm{min}$ and oxygen saturation of $90 \%$ on room air at rest. Patient in apparent fair general condition. Pain and crackles on palpation in the anterior face of the neck, in relation to subcutaneous emphysema. Rest of the examination without significant abnormalities. Hemogram on admission showed leukocytosis without shift to the left. (11000 leukocytes with $4 \%$ of bands). The lung x-ray did not show pneumonic involvement, but it did show subcutaneous emphysema and pneumomediastinum.

Given the findings, an evaluation was requested by Intensive Care who admitted her to their service 6 hours after the beginning of the clinical picture. Antibiotic treatment was started with Vancomycin 500mg every 12 hours and Meropenem 1g every 8 hours.

At 12 hours, the patient presented a thermal rise of $37.5^{\circ} \mathrm{C}$ and a significant increase in subcutaneous emphysema and later respiratory distress (Figure 1). Inotropic drugs were started for an episode of hypotension (BP: 80/50 $\mathrm{mmHg}$ ) and oxygen therapy with non-invasive ventilation.

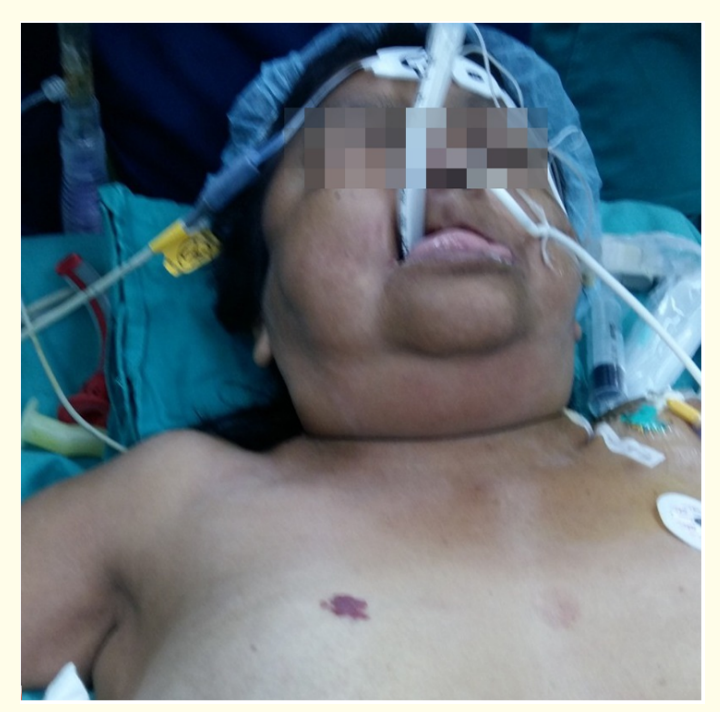

Figure 1: Extensive subcutaneous emphysema on the face, neck and chest.

A chest tomography with water-soluble contrast was performed at 18 hours, where a mediastinal collection was demonstrated. With these findings, a thoracic surgery and general surgery consultation was requested (Figures 2 and 3).

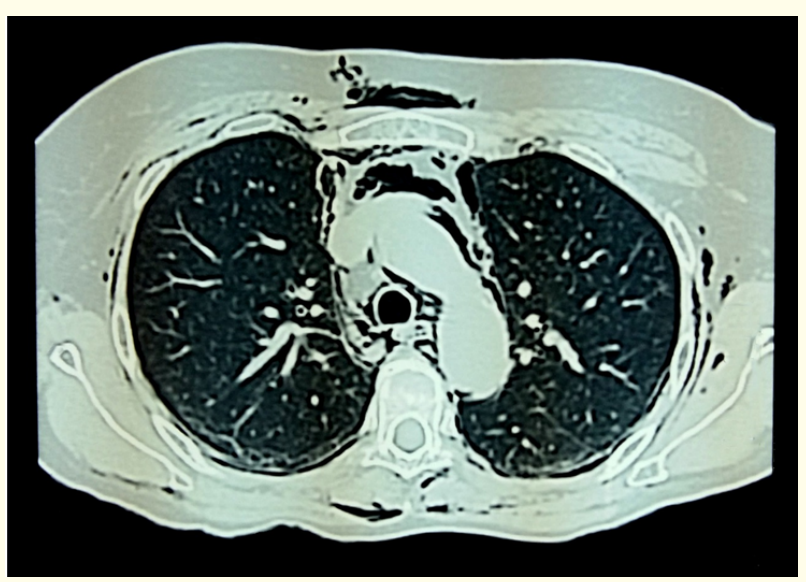

Figure 2: Computed tomography showing pneumomediastinum and the presence of a collection. Laminar pneumothorax and extensive subcutaneous emphysema in the chest.

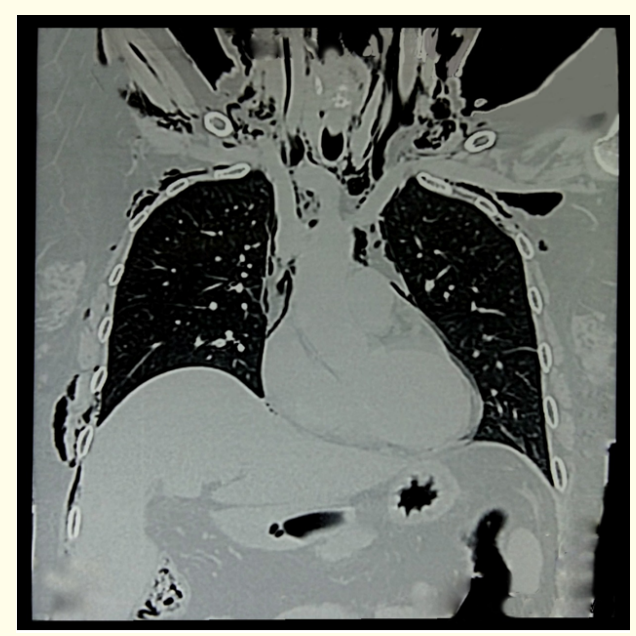

Figure 3: Chest tomography shows the presence of a collection in the mediastinum, laminar pneumothorax and extensive subcutaneous emphysema in the chest.

At 22 hours a medical meeting was held to determine surgical treatment as soon as possible, and the plan was: extensive mediastinal debridement + bilateral drainage tube by thoracoscopic surgery + jejunostomy. The intervention had no intercurrences and the patient was hemodynamically stable.

To avoid the collection of saliva at the gastric level, continuous suction through a nasogastric tube was chosen. 
The patient was kept in the critical care unit for seven days, during which she was given enteral nutrition by jejunostomy, and continuous suction of saliva by nasogastric tube. She was discharged in the second week. There were no complications during follow-up.

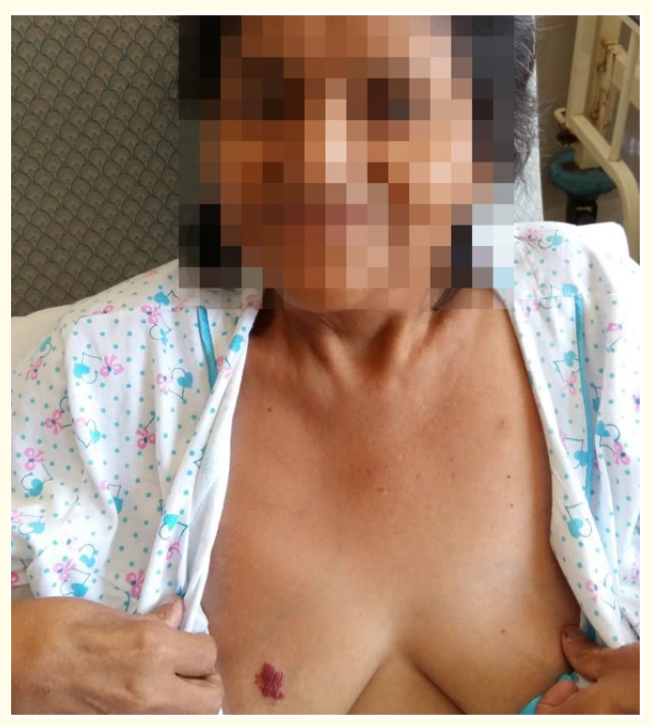

Figure 5: $7^{\text {th }}$ day post operative.

\section{Discussion and Conclusion}

Since the esophageal wall is thin, it is an organ that can be easily injured. Endoscopic esophageal dilation procedures can cause its perforation. A late diagnosis and treatment (more than 24 hours after the symptoms started) give a mortality that can easily exceed $50 \%$. Sudden onset of pain after endoscopic intervention is the most common symptom [1-4]. In the case of our patient, he presented anterosuperior chest pain after esophageal dilation.

Contrast computed tomography (CT) is the imaging test of choice in patients with suspected esophageal perforation. CT helps to assess extension to adjacent structures (accumulation of air or fluid in the mediastinum, pleural effusions) and to guide initial therapy. The presence of mediastinal fluid, specifically within the visceral compartment, strongly suggests an esophageal injury, and its absence gives a negative predictive value [6].

Diagnostic endoscopy is useful in patients with suspected PE and doubtful findings on CT. Diagnostic endoscopy for PD is reli- able and safe in experienced hands; however, the potential risks of enlarging the size of the perforation and increasing contamination of the surrounding spaces warrant caution and limit its use as a first-line examination $[1,2]$.

As evidenced in the case of our patient, the presence of fluid in the mediastinum was evidenced, which led to admission to the surgical room for bilateral debridement of the mediastinum by thoracoscopic surgery (VATS) and placement of drainage tubes.

This patient started with a systemic inflammatory response, so it was decided to prefer drainage and extensive debridement over primary closure. Two reasons motivated drainage and debridement without primary closure, the first is that VATS drainage is within aggressive nonoperative management, and the second is that the initial etiology is a peptic stricture with scar tissue that makes primary closure impossible [4]. Management without primary closure of the esophagus is safe and effective for early perforation $(<24$ hours) [1,2]. In all cases, the mediastinum should be left wide open bilaterally and necrotic tissues should be debrided. The surgical approach must be adapted to the particular clinical situation of the patient and the surgical experience of the surgeon [7]. Delaying diagnosis and treatment beyond 24 hours is associated with a higher mortality rate $[8,9]$.

Patients with esophageal perforation should be kept to nothing by mouth, administration of broad-spectrum antibiotics (aerobic and anaerobic bacteria), and therapy with proton pump inhibitors. Early introduction of nutritional support through enteral feeding or total parenteral nutrition is essential for healing of the esophagus. Endoscopic placement of a nasogastric tube is recommended [1].

The Pittsburgh Severity Score (PSS) classification has been developed to include an esophageal perforation score based on ten clinical and radiological factors to assist PD patients with decisionmaking [1].

The PSS is a clinical score based on pre-existing esophageal pathology and clinical findings at presentation. All variables are assigned points (range, 1-3) for a possible total score of 18 . Points are awarded to each variable according to the following scale: 1 $=$ age $>75$ years, tachycardia $(>100 \mathrm{bpm})$, leukocytosis $(>10,000$ white blood cells/ml) or pleural effusion (on chest x-ray, CT scan, 
or esophagram); 2 = fever $\left(>38.5^{\circ} \mathrm{C}\right)$, uncontained leak (on esophagogram or CT scan), respiratory compromise (respiratory rate $>30$, increased need for oxygen or need for mechanical ventilation), or time to diagnosis> 24 hours; and 3 = presence of cancer or hypotension. The score has been validated in a multinational study and it has been suggested that patients with a low score $(\leq 2)$ could be eligible for non-surgical treatment and are considered low risk. A score of 3 to 5 is intermediate risk, and a score greater than 5 is classified as high risk. In the latter case, extensive surgical management predominates [5].

Our patient presented a severity score of 6, that is, high risk, and debridement and drainage were performed with large-caliber chest tubes.

In conclusion, this case shows that the suspected diagnosis of perforation after an esophageal dilation procedure is key for timely treatment (less than 24 hours). Management of esophageal perforation with critical care and aggressive management (drainage plus extensive debridement of the mediastinum by VATS) obtained a good result.

\section{Bibliography}

1. Chirica M., et al. "Esophageal emergencies: WSES guidelines". World Journal of Emergency Surgery 14 (2019): 26.

2. Puerta Vicente A., et al. "Management of Esophageal Perforation: 28-Year Experience in a Major Referral Center". American Surgery 84.5 (2018): 684-689.

3. Tullavardhana T. "Iatrogenic Esophageal Perforation". Journal of the Medical Association of Thailand 98.9 (2015): S177-183.

4. Skinner MJ and Thompson CC. "Management of perforation following stricture dilation". Minerva Chirurgica 72.4 (2017): 347-360.

5. Wigley C., et al. "Does the Pittsburgh Severity Score predict outcome in esophageal perforation?" Diseases of the Esophagus 32.2 (2017).

6. Fuhrmann C., et al. "Mediastinal fluid as a predictor for esophageal perforation as the cause of pneumomediastinum". Emergency Radiology 28.2 (2021): 233-238.

7. Eroglu A., et al. "Thoracic perforations-surgical techniques". Annals of Translational Medicine's 6.3 (2018): 40.
8. Sdralis EIK., et al. "Epidemiology, diagnosis, and management of esophageal perforations: systematic review". Diseases of the Esophagus 30.8 (2017): 1-6.

9. Krüger M., et al. "Therapie der akuten Mediastinitis [Surgical treatment of acute mediastinitis]". Chirurg 87.6 (2016): 478485.

\section{Volume 4 Issue 12 December 2021}

(C) All rights are reserved by Castillo De La Cadena Luis. 